\title{
On the quermassintegrals of convex bodies
}

\author{
Chang Jian Zhao ${ }^{1 *}$ and Wing Sum Cheung ${ }^{2}$
}

*Correspondence:

chjzhao@163.com;

chjzhao@yahoo.com.cn

${ }^{1}$ Department of Mathematics, China

Jiliang University, Hangzhou,

310018, P.R. China

Full list of author information is

available at the end of the article

\section{Springer}

\section{Abstract}

The well-known question for quermassintegrals is the following: For which values of $i \in \mathbb{N}$ and every pair of convex bodies $K$ and $L$, is it true that

$$
\frac{W_{i}(K+L)}{W_{i+1}(K+L)} \geq \frac{W_{i}(K)}{\tilde{W}_{i+1}(K)}+\frac{W_{i}(L)}{W_{i+1}(L)} ?
$$

In 2003, the inequality was proved if and only if $i=n-1$ or $i=n-2$. Following the problem, in the paper, we prove some interrelated results for the quermassintegrals of a convex body.

MSC: $26 \mathrm{D} 15 ; 52 \mathrm{~A} 30$

Keywords: symmetric function; convex body; quermassintegral

\section{Introduction}

The origin of this work is an interesting inequality of Marcus and Lopes [1]. We write $E_{i}(x), 0 \leq i \leq n$, for the $i$ th elementary symmetric function of an $n$-tuple $x=\left(x_{1}, \ldots, x_{n}\right)$ of positive real numbers. This is defined by $E_{0}(x)=1$ and

$$
E_{i}(x)=\sum_{1 \leq j_{1}<\cdots<i_{i} \leq n} x_{j_{1}} x_{j_{2}} \cdots x_{j_{i}}, \quad 1 \leq i \leq n
$$

In particular, $E_{1}(x)=x_{1}+\cdots+x_{n}, E_{2}(x)=\sum_{i \neq j} x_{i} x_{j}, \ldots, E_{n}(x)=x_{1} x_{2} \cdots x_{n}$.

The Marcus-Lopes inequality (see also [2, p.33]) states that

$$
\frac{E_{i}(x+y)}{E_{i-1}(x+y)} \geq \frac{E_{i}(x)}{E_{i-1}(x)}+\frac{E_{i}(y)}{E_{i-1}(y)}
$$

for every pair of positive $n$-tuples $x$ and $y$. This is a refinement of a further result concerning the symmetric functions, namely

$$
\left[E_{i}(x+y)\right]^{1 / i} \geq\left[E_{i}(x)\right]^{1 / i}+\left[E_{i}(y)\right]^{1 / i}
$$

A discussion of the cases of equality is contained in the reference [1].

A matrix analogue of (1.1) is the following result of Bergstrom [3] (see also the article [4] and [5, p.67] for an interesting proof): If $K$ and $L$ are positive definite matrices, and if $K_{i}$ and $L_{i}$ denote the submatrices obtained by deleting their $i$ th row and column, then

$$
\frac{\operatorname{det}(K+L)}{\operatorname{det}\left(K_{i}+L_{i}\right)} \geq \frac{\operatorname{det}(K)}{\operatorname{det}\left(K_{i}\right)}+\frac{\operatorname{det}(L)}{\operatorname{det}\left(L_{i}\right)} .
$$


The following generalization of (1.3) was established by Ky Fan [5]:

$$
\left(\frac{\operatorname{det}(K+L)}{\operatorname{det}\left(K_{i}+L_{i}\right)}\right)^{1 / k} \geq\left(\frac{\operatorname{det}(K)}{\operatorname{det}\left(K_{i}\right)}\right)^{1 / k}+\left(\frac{\operatorname{det}(L)}{\operatorname{det}\left(L_{i}\right)}\right)^{1 / k}
$$

The proof is based on a minimum principle; see also Ky Fan [6] and Mirsky [7].

There is a remarkable similarity between inequalities about symmetric functions (or determinants of symmetric matrices) and inequalities about the mixed volumes of convex bodies. For example, the analogue of (1.2) in the Brunn-Minkowski theory is as follows.

If $K$ and $L$ are convex bodies in $\mathbb{R}^{n}$ and if $0 \leq i \leq n-1$, then

$$
W_{i}(K+L)^{1 /(n-i)} \geq W_{i}(K)^{1 /(n-i)}+W_{i}(L)^{1 /(n-i)},
$$

with equality if and only if $K$ and $L$ are homothetic, where $W_{i}(K)$ is the $i$ th quermassintegral of $K$ (see Section 2).

In view of this analogue, Milman asked if there exists a version of (1.1) or (1.3) in the theory of mixed volumes (see $[8,9])$.

Question For which values of $0 \leq i \leq n-1, i \in \mathbb{N}$, is it true that, for every pair of convex bodies $K$ and $L$ in $\mathbb{R}^{n}$, one has

$$
\frac{W_{i}(K+L)}{W_{i+1}(K+L)} \geq \frac{W_{i}(K)}{W_{i+1}(K)}+\frac{W_{i}(L)}{W_{i+1}(L)} ?
$$

In 1991, the special case $i=0$ was stated also in [10] as an open question. In the same paper it was also mentioned that (1.6) follows directly from the Aleksandrov-Fenchel inequality when $i=0$ and $L$ is a ball.

In 2002, it was proved in [9] that (1.6) is true for all $i=1, \ldots, n-1$ in the case where $L$ is a ball.

Theorem A If $K$ is a convex body and $B$ is a ball in $\mathbb{R}^{n}$, then for $0 \leq i \leq n-1, i \in \mathbb{N}$,

$$
\frac{W_{i}(K+B)}{W_{i+1}(K+B)} \geq \frac{W_{i}(K)}{W_{i+1}(K)}+\frac{W_{i}(B)}{W_{i+1}(B)} .
$$

In 2003, it was proved in [8] that (1.6) holds true for every pair of convex bodies $K$ and $L$ in $\mathbb{R}^{n}$ if and only if $i=n-2$ or $i=n-1$.

Theorem B Let $0 \leq i \leq n-1$, then

$$
\frac{W_{i}(K+L)}{W_{i+1}(K+L)} \geq \frac{W_{i}(K)}{W_{i+1}(K)}+\frac{W_{i}(L)}{W_{i+1}(L)}
$$

is true for every pair of convex bodies $K$ and $L$ in $\mathbb{R}^{n}$ if and only if $i=n-1$ or $i=n-2$.

In this paper, following the above results, we prove the following interest results.

Theorem 1.1 Let $0 \leq i \leq n-1$ and for every convex body $K$ and $L$ in $\mathbb{R}^{n}$. Then the function

$$
g(t)=\frac{W_{i}(K+t L)}{W_{i+1}(K+t L)}
$$

is a convex function on $t \in[0,+\infty)$ if and only if $i=n-1$ or $i=n-2$. 
Theorem 1.2 Let $0 \leq i \leq n-1$ and for every convex body $K$ and $L$ in $\mathbb{R}^{n}$. Then

$$
\begin{aligned}
& (n-i) W_{i+2}(K)\left(W_{i+1}(K)^{2}-W_{i}(K) W_{i+2}(K)\right) \\
& \quad \geq(n-i-2) W_{i}(K)\left(W_{i+2}^{2}(K)-W_{i+1}(K) W_{i+3}(K)\right)
\end{aligned}
$$

if and only if $i=n-1$ or $i=n-2$.

\section{Notations and preliminaries}

The setting for this paper is an $n$-dimensional Euclidean space $\mathbb{R}^{n}$. Let $\mathcal{K}^{n}$ denote the set of convex bodies (compact, convex subsets with non-empty interiors) in $\mathbb{R}^{n}$. We reserve the letter $u$ for unit vectors, and the letter $B$ for the unit ball centered at the origin. The surface of $B$ is $S^{n-1}$. The volume of the unit $n$-ball is denoted by $\omega_{n}$.

We use $V(K)$ for the $n$-dimensional volume of a convex body $K$. Let $h(K, \cdot): S^{n-1} \rightarrow \mathbb{R}$ denote the support function of $K \in \mathcal{K}^{n}$; i.e., for $u \in S^{n-1}$,

$$
h(K, u)=\operatorname{Max}\{u \cdot x: x \in K\},
$$

where $u \cdot x$ denotes the usual inner product $u$ and $x$ in $\mathbb{R}^{n}$.

Let $\delta$ denote the Hausdorff metric on $\mathcal{K}^{n}$, i.e., for $K, L \in \mathcal{K}^{n}, \delta(K, L)=\left|h_{K}-h_{L}\right|_{\infty}$, where $|\cdot|_{\infty}$ denotes the sup-norm on the space of continuous functions $C\left(S^{n-1}\right)$.

Associated with a compact subset $K$ of $\mathbb{R}^{n}$, which is star-shaped with respect to the origin, is its radial function $\rho(K, \cdot): S^{n-1} \rightarrow \mathbb{R}$, defined for $u \in S^{n-1}$ by

$$
\rho(K, u)=\operatorname{Max}\{\lambda \geq 0: \lambda u \in K\} .
$$

If $\rho(K, \cdot)$ is positive and continuous, $K$ will be called a star body. Let $\mathcal{S}^{n}$ denote the set of star bodies in $\mathbb{R}^{n}$. Let $\tilde{\delta}$ denote the radial Hausdorff metric, as follows, if $K, L \in \mathcal{S}^{n}$, then $\tilde{\delta}(K, L)=\left|\rho_{K}-\rho_{L}\right|_{\infty}$.

If $K_{i} \in \mathcal{K}^{n}(i=1,2, \ldots, r)$ and $\lambda_{i}(i=1,2, \ldots, r)$ are nonnegative real numbers, then of fundamental importance is the fact that the volume of $\sum_{i=1}^{r} \lambda_{i} K_{i}$ is a homogeneous polynomial in the $\lambda_{i}$ given by (see, e.g., [11] or [12])

$$
V\left(\lambda_{1} K_{1}+\cdots+\lambda_{n} K_{n}\right)=\sum_{i_{1}, \ldots, i_{n}} \lambda_{i_{1}} \cdots \lambda_{i_{n}} V_{i_{1}, \ldots, i_{n}}
$$

where the sum is taken over all $n$-tuples $\left(i_{1}, \ldots, i_{n}\right)$ of positive integers not exceeding $r$. The coefficient $V_{i_{1}, \ldots, i_{n}}$ depends only on the bodies $K_{i_{1}}, \ldots, K_{i_{n}}$ and is uniquely determined by (2.1). It is called the mixed volume of $K_{i_{1}}, \ldots, K_{i_{n}}$, and is written as $V\left(K_{i_{1}}, \ldots, K_{i_{n}}\right)$. Let $K_{1}=$ $\cdots=K_{n-i}=K$ and $K_{n-i+1}=\cdots=K_{n}=L$, then the mixed volume $V\left(K_{1}, \ldots, K_{n}\right)$ is written as $V_{i}(K, L)$. If $K_{1}=\cdots=K_{n-i}=K, K_{n-i+1}=\cdots=K_{n}=B$, then the mixed volume $V_{i}(K, B)$ is written as $W_{i}(K)$ and is called the quermassintegral of a convex body $K$.

It is convenient to write relation (2.1) in the form (see [12, p.137])

$$
\begin{aligned}
& V\left(\lambda_{1} K_{1}+\cdots+\lambda_{s} K_{s}\right) \\
& \quad=\sum_{p_{1}+\cdots+p_{r}=n} \sum_{1 \leq i_{1}<\cdots<i_{r} \leq s} \frac{n !}{p_{1} ! \cdots p_{r} !} \lambda_{i_{1}}^{p_{1}} \cdots \lambda_{i_{r}}^{p_{r}} V(\underbrace{K_{i_{1}}, \ldots, K_{i_{1}}}_{p_{1}}, \ldots, \underbrace{K_{i_{r}}, \ldots, K_{i_{r}}}_{p_{r}}) .
\end{aligned}
$$


Let $s=2, \lambda_{1}=1, K_{1}=K, K_{2}=B$, we have

$$
V(K+\lambda B)=\sum_{i=0}^{n}\left(\begin{array}{l}
n \\
i
\end{array}\right) \lambda^{i} W_{i}(K)
$$

known as formula 'Steiner decomposition'.

On the other hand, for convex bodies $K$ and $L$, (2.2) can show the following special case:

$$
W_{i}(K+\lambda L)=\sum_{j=0}^{n-i}\left(\begin{array}{c}
n-i \\
j
\end{array}\right) \lambda^{j} V(\underbrace{K, \ldots, K}_{n-i-j}, \underbrace{B, \ldots, B}_{i}, \underbrace{L, \ldots, L}_{j}) .
$$

\section{Proof of main results}

Proof of Theorem 1.1 If $s, t \in[0, \infty)$, from (1.8), if and only if $i=n-1$ or $i=n-2$, we have

$$
\begin{aligned}
g\left(\frac{t+s}{2}\right) & =\frac{W_{i}\left(K+\frac{t+s}{2} L\right)}{W_{i+1}\left(K+\frac{t+s}{2} L\right)} \\
& =\frac{W_{i}\left(\frac{K+t L}{2}+\frac{K+s L}{2}\right)}{W_{i+1}\left(\frac{K+t L}{2}+\frac{K+s L}{2}\right)} \\
& \geq \frac{W_{i}\left(\frac{K+t L}{2}\right)}{W_{i+1}\left(\frac{K+t L}{2}\right)}+\frac{W_{i}\left(\frac{K+s L}{2}\right)}{W_{i+1}\left(\frac{K+s L}{2}\right)} \\
& =\frac{1}{2} \frac{W_{i}(K+t L)}{W_{i+1}(K+t L)}+\frac{1}{2} \frac{W_{i}(K+s L)}{W_{i+1}(K+s L)} \\
& =\frac{1}{2}(g(t)+g(s)) .
\end{aligned}
$$

Hence the function $g(t)$ is a convex function on $[0,+\infty)$ for every star body $K$ and $L$ if and only if $i=n-1$ or $i=n-2$.

Proof of Theorem 1.2 Let $K$ be a convex body in $\mathbb{R}^{n}$. For every $i \geq 0$, we set

$$
f_{i}(t)=W_{i}(K+t B)
$$

then from (2.3)

$$
\begin{aligned}
f_{i}(t+\varepsilon) & =W_{i}((K+t B)+\varepsilon B) \\
& =\sum_{j=0}^{n-i}\left(\begin{array}{c}
n-i \\
j
\end{array}\right) \varepsilon^{j} W_{i+j}(K+t B) \\
& =f_{i}(t)+\varepsilon(n-i) f_{i+1}(t)+O\left(\varepsilon^{2}\right) .
\end{aligned}
$$

Therefore

$$
f_{i}^{\prime}(t)=(n-i) f_{i+1}(t)
$$

The derivative of the function

$$
g_{i}(t)=\frac{f_{i}(t)}{f_{i+1}(t)}=\frac{W_{i}(K+t B)}{W_{i+1}(K+t B)}
$$


is thus given by

$$
g_{i}^{\prime}(t)=(n-i)-(n-i-1) \frac{f_{i}(t) f_{i+2}(t)}{f_{i+1}^{2}(t)} .
$$

Since $g_{i}(x)$ is a convex function if and only if $i=n-1$ or $i=n-2$, hence by differentiating the both sides of (3.2), we obtain for $t \in(0,+\infty)$

$$
(n-i) f_{i+2}(t) f_{i+1}^{2}(t)+(n-i-2) f_{i}(t) f_{i+1}(t) f_{i+3}(t)-2(n-i-1) f_{i}(t) f_{i+2}^{2}(t) \geq 0
$$

if and only if $i=n-1$ or $i=n-2$.

This can be equivalently written in the form

$$
(n-i) f_{i+2}(t)\left(f_{i+1}^{2}(t)-f_{i}(t) f_{i+2}(t)\right) \geq(n-i-2) f_{i}(t)\left(f_{i+2}^{2}(t)-f_{i+1}(t) f_{i+3}(t)\right)
$$

if and only if $i=n-1$ or $i=n-2$.

Letting $t \rightarrow 0^{+}$, we conclude Theorem 1.2.

\section{Competing interests}

The authors declare that they have no competing interests.

\section{Authors' contributions}

CIZ and WSC jointly contributed to the main results Theorems 1.1-1.2. All authors read and approved the final manuscript.

\section{Author details}

${ }^{1}$ Department of Mathematics, China Jiliang University, Hangzhou, 310018, P.R. China. ${ }^{2}$ Department of Mathematics, The University of Hong Kong, Pokfulam Road, Hong Kong, P.R. China.

\section{Acknowledgements}

First author is supported by the National Natural Science Foundation of China (10971205). Second author is partially supported by a HKU URG grant.

\section{Received: 18 March 2013 Accepted: 9 May 2013 Published: 27 May 2013}

\section{References}

1. Marcus, M, Lopes, I: Inequalities for symmetric functions and Hermitian matrices. Can. J. Math. 8, 524-531 (1956)

2. Bechenbach, EF, Bellman, R: Inequalities. Springer, Berlin (1961)

3. Bergstrom, H: A triangle inequality for matrices. In: Den Elfte Skandinaviski Matematiker-kongress, Trondheim, 1949. John Grundt Tanums Forlag, Oslo (1952)

4. Bellman, R: Notes on matrix theory - IV: an inequality due to Bergstrom. Am. Math. Mon. 62, 172-173 (1955)

5. Fan, K: Some inequalities concerning positive-definite Hermitian matrices. Proc. Camb. Philos. Soc. 51, 414-421 (1955)

6. Fan, K: Problem 4786. Am. Math. Mon. 65, 289 (1958)

7. Mirsky, L: Maximum principles in matrix theory. Proc. Glasg. Math. Assoc. 4, 34-37 (1958)

8. Fradelizi, M, Giannopoulos, A, Meyer, M: Some inequalities about mixed volumes. Isr. J. Math. 135, 157-179 (2003)

9. Giannopoulos, A, Hartzoulaki, M, Paouris, G: On a local version of the Aleksandrov-Fenchel inequality for the quermassintegrals of a convex body. Proc. Am. Math. Soc. 130, 2403-2412 (2002)

10. Dembo, A, Cover, TM, Thomas, JA: Information theoretic inequalities. IEEE Trans. Inf. Theory 37, 1501-1518 (1991)

11. Schneider, R: Convex Bodies: The Brunn-Minkowski Theory. Cambridge University Press, Cambridge (1993)

12. Burago, YD, Zalgaller, VA: Geometric Inequalities. Springer, Berlin (1988)

doi:10.1186/1029-242X-2013-264

Cite this article as: Zhao and Cheung: On the quermassintegrals of convex bodies. Journal of Inequalities and Applications 2013 2013:264. 